\title{
EFEITOS DE GEADAS EM MUDAS DE ESPÉCIES ARBÓREAS DE MATA CILIAR UTILIZADAS EM ENSAIOS DE CAMPO. ${ }^{1}$
}

\author{
Luiz Mauro Barbosa'; \\ José Marcos Barbosa; \\ Cristina Bedinelli; \\ Lilian Maria Asperti ${ }^{3}$; \\ Evelyn de Freitas Belasque ${ }^{3}$ \\ Eduardo Amaral Batista ${ }^{4}$.
}

Recebido em 16-09-91. Aceito em 24-05-93

\begin{abstract}
RESUMO - Indivíduos de espécies arbóreas ocorrentes em matas ripárias, plantadas com idades entre 1 e 2 anos, foram investigadas quanto à sensibilidade a geadas a partir de junho/agosto de 1990, quando estas ocorreram no município de Moji-Guaçu, SP. Dessas, pelo menos duas atingiram as mudas na área experimental. Cerca de 2.750 indivíduos de 13 espécies, plantados numa área de 2,5 ha, situada à margem direita do rio Moji-Guaçu, foram avaliados mensalmente quanto à variação de altura total e dos seus rebrotes. Atribuíram-se notas de 1 a 5 , conforme critérios pré-estabelecidos e observaram-se: aspectos da morfologia geral; início do brotamento das gemas vegetativas e o comportamento dos indivíduos de acordo com a proximidade do rio. Os efeitos mais evidentes nas plantas foram: modificação da cor e textura das folhas, que ficaram enegrecidas ou avermelhadas, seguida de abscisão e morte total ou parcial da parte aérea. Os resultados indicam que das espécies estudadas, apenas Platycyamus regnelli Benth. (Leguminosae), Hymenaea courbaril L. (Leguminosae) e Alchornea sp. (Euphorbiaceae), apresentaram restriçōes quanto ao plantio por mudas, em regiōes sujeitas à geadas periódicas. As demais espécies demonstraram grande capacidade de recuperação, sugerindo resistência e/ou tolerância, quando sob ação de geadas.
\end{abstract}

Palavras-chave: floresta ripária, geada, espécies nativas.

ABSTRACT - One to two-year-old seedlings of tree species found in riparian forests were investigated for frost sensibility as of June/August 1990, in MojiGuaçu Municipality.São Paulo State. At least two frost affected the seedlings

1 - Trabalho apresentado no XLII Congresso Nacional de Botânica, Goiânia-GO.

2. Instituto de Botânica de São Paulo. Cx. P. 4005, CEP 0430-012, SP. Bolsista do CNPq.

3 . Estagiárias do Instituto de Botânica de São Paulo. Bolsistas do CNPq.

4 . Instituto Florestal do Estado de São Paulo. 
in the experimental area during this period. Around 2750 seedlings from 13 species were planted in a 2.5-hectare area on the shores of the Moji-Guaçu river. Variations in total height and budding were recorded monthly. Scores from to 5 were given based on pre-stablished criteria. General morphology, initiation of vegetative growth and seedling behavior were observed in relation to riverbank proximity. The most obvious effects on seedlings were changes in leaf color and texture; the leaves turned blackish or reddish, followed by abscission and total or partial death of aerial parts. Of the species investigated, planting restrictions on seedlings in frost-prone areas should be applied only to Platycyamus regnelli Benth. (Leguminosae), Hymenaea courbaril L. (Leguminosae) and Alchornea s (Euphorbiaceae). The other species showed considerable capacity for recuperation, suggesting resistance and/or tolerance to frost damage.

Key words: riparian forest, frost, native species.

\section{Introdução}

0 presente trabalho foi desenvolvido em função da ocorrência de geadas entre os meses de junho a agosto de 1990, na região de Moji-Guaçu (SP), onde vem sendo desenvolvida parte das investigações de um projeto integrado, que visa a implantação e análise de modelos para conservação e recuperação de áreas degradadas, em margens de cursos d'água. 0 projeto inclui estudos fitossociológicos, ecofisiológicos e de tecnologia de sementes das espécies de ampla ocorrência em matas ciliares, cujos primeiros resultados foram pulicados por Barbosa et al. (1989a) e Barbosa et al. (1989b).

Vários autores fizeram consideraçōes sobre a importância das matas ciliares, ripárias ou de galerias, especialmente na proteção contra assoreamento que ocorre em função de diversos tipos de erosão, ou na interferência sobre o microclima regional e na dinâmica de populações da fauna regional (Marques et al., 1961; Porto et al., 1976; Salvador, 1987; Barbosa, 1989; Demattê, 1989 e Mantovani, 1989).

A literatura referente às plantas tropicais é farta em informações técnico-científico sobre os danos causados por geadas às espécies cultivadas, contudo, pouco se sabe a respeito dos efeitos desse fenômeno meteorológico sobre as comunidades vegetais naturais, especialmente no Brasil (Struffaldi De-Vuono et al., 1982), podendo se destacar o trabalho de SilberbauerGottsberger et al. (1977), onde os autores realizaram alguns estudos sobre os efeitos da geada em plantas de cerrado.

Neste trabalho são apresentadas informações sobre o comportamento de 13 espécies típicas de mata ciliar sob o efeito de geadas, na região de Moji-Guaçu (SP). 


\section{Material e Métodos}

O presente estudo vem sendo desenvolvido em área urbana do Município de Moji-Guaçu, à margem direita do rio de mesmo nome, nas coordenadas $22^{\circ} 18^{\prime} \mathrm{S}$ e $47^{\circ} 11^{\prime} 0$, e altitude média de 680 metros. 0 clima da região é do tipo Cwa, segundo a classificação de Köppen, com inverno seco e temperatura média acima de $22^{\circ} \mathrm{C}$, no mês mais quente, e $18^{\circ} \mathrm{C}$, no mês mais frio.

Os valores de temperaturas máxima, média e mínima e de precipitação média nos meses de junho a agosto de 1990, foram obtidos na Estação Meteorológica da Fazenda Campininha, localizada no Município de Moji-Guaçu, onde está situada a área experimental. Os dados são apresentados na Figura 1, cuja temperatura mínima absoluta nos dias $24 / 06$ e 30/07, quando possivelmente foram causados os principais danos das geadas, atingiram $0,6^{\circ} \mathrm{C}$ e $3,0^{\circ} \mathrm{C}$, respectivamente.

Tais informações não descartam a possibilidade de que na microregião, ou seja, no local de instalação dos ensaios, a temperatura possa ter atingido valores negativos.

A área do experimento é de aproximadamente 2,5 ha, na qual foram plantadas (desde dezembro/1987) 2.750 mudas de 13 espécies típicas de mata ripária, com idades variáveis entre 1 e 2 anos. A quantidade de mudas utilizadas por espécies nos plantios variou de acordo com a disponibilidade de sementes e mudas.

Contudo, as avaliações ocorreram sempre de forma proporcional ao número de indivíduos utilizados. As 13 espécies e respectivos números de indivíduos estudados nesta pesquisa foram: Alchornea sp. (Euphorbiaceae) - 71; Cariniana sp. (Lecythydaceae) - 317; Copaifera langsdorfii Desf. (Leguminosae) - 215; Cordia sp. (Boraginaceae) - 353; Croton urucurana Baill. (Euphorbiaceae) - 68; Cyclolobium vecchii A Samp ex Hoehne (Leguminosae) - 225; Enterolobium contortisiliquum (Vell.) Morong (Leguminosae) - 94; Erythrina cristagalli L. (Leguminosae) - 21; Genipa americana L. (Rubiaceae) - 353; Hymenaea courbaril L. (Leguminosae) 102; Inga striata Willd. (Leguminosae) - 624; Lonchocarpus muehlbergianus Hassl. (Leguminosae) - 131 e Platycyamus regnelli Benth. (Leguminosae) - 62..

As mudas encontram-se dispostas em 83 linhas perpendiculares ao rio, com uma média de 33 mudas por linha, em espaçamento de $3 \times 3 \mathrm{~m}$. A área do experimento foi dividida em 4 faixas em relação ao rio (faixa $1=0-21 \mathrm{~m}$; faixa $2=$ 21-42m; faixa $3=42-63 \mathrm{~m}$ e faixa $4=$ mais de $63 \mathrm{~m}$ da margem do rio) (figura 2 ).

A partir de agosto, um mês após a ocorrência da última geada da área, foram realizadas avaliações mensais durante 4 meses, quando se verificou: números de indivíduos de cada espécie, por faixa, e suas respectivas alturas; incremento em altura; início de brotamento de gemas vegetativas e suas alturas, além dos efeitos da geada através de observaçöes visuais no campo, atribuindo-se notas aos indivíduos, que variaram de 1 a 5 , conforme critérios pré-estabelecidos $(1=$ morta; 2 = muito afetada; 3 = mediante afetada; 4 = pouco afetada e 5 = não afetada pela geada). 

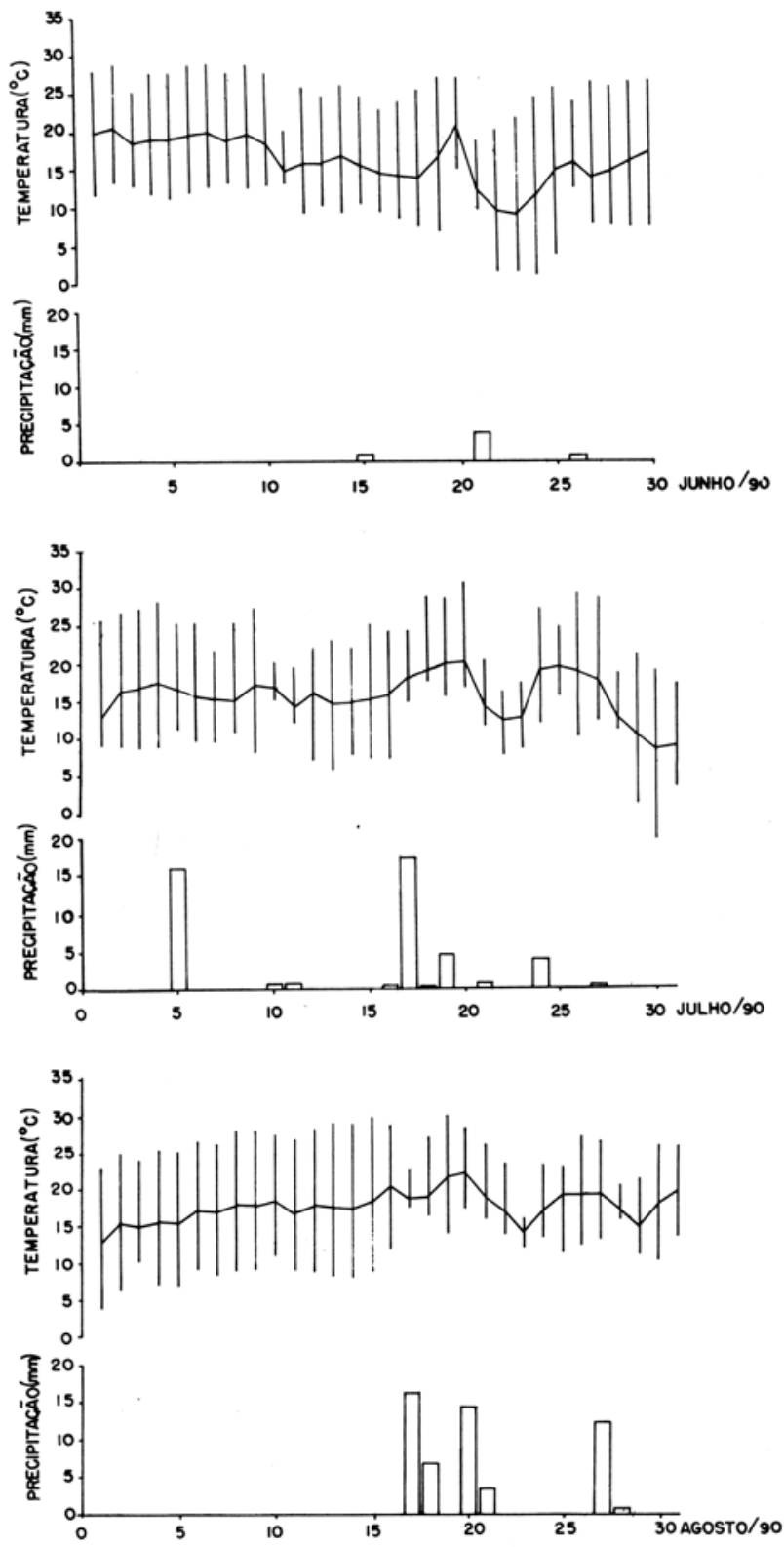

Figura 1 - Média de temperatura, amplitude de variação diária e precipitação total diária nos meses de junho a agosto de 1990, registradas no posto meteorológico da Fazenda Campininha, Moji-Guaçu (SP).

(Temperaturas médias de $16,07^{\circ} \mathrm{c} ; 5,77^{\circ} \mathrm{c}$ e $17,61^{\circ} \mathrm{c}$ e, precipitaçöes totais de 5,$6 ; 45,9$ e $55,5 \mathrm{~mm}$ nos meses de junho e agosto de 1990 , respectivamente). 


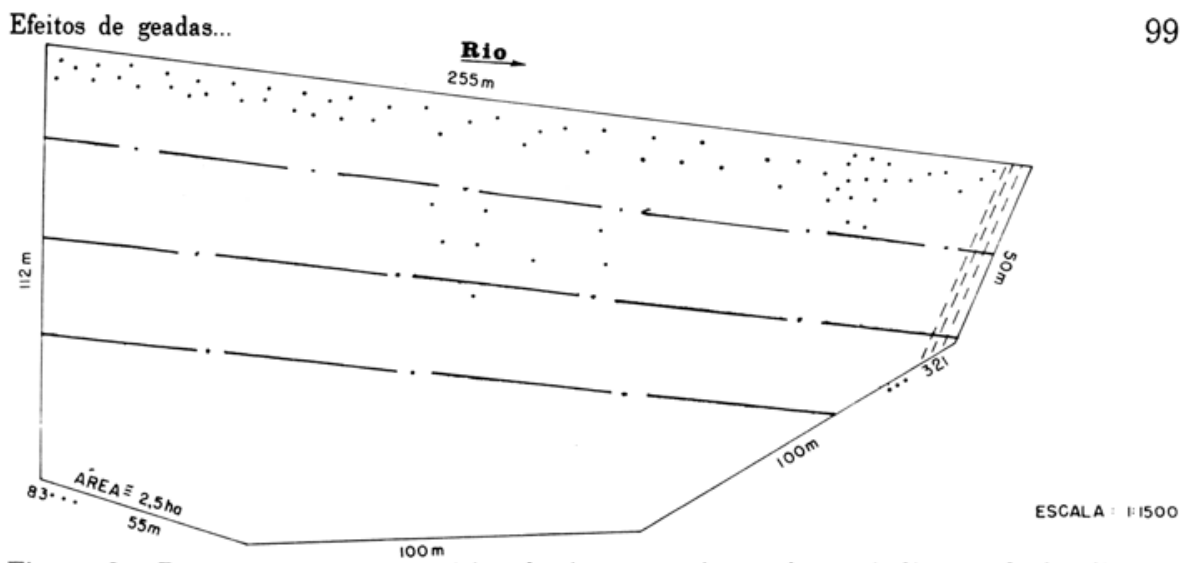

Figura 2 - Representaçảo esquemática da área experimental com indicação da localização dos indivíduos adultos remanescentes na área e locação das linhas de plantio, do rio e das quatro faixas de amostragem $(0-21 ; 21-42 ; 42-63$ e maior de 63 metros da margem do rio). Moji-Guaçu - 1990.

Os dados obtidos foram lançados em planilha de cálculo do Programa Totalworks, em computador TK 3000, para a obtenção dos parâmetros indicativos do grau de interferência da geada sobre os indivíduos de cada espécie por faixa (nota média dos indivíduos, porcentagem média de mortalidade e altura média de cada espécie estudada nesta pesquisa)

\section{Resultados e discussão}

O sintoma mais evidente nas plantas afetadas foi a modificação da cor e textura das folhas que se tornaram friáveis e escuras, seguindo-se a abscisão. A variação da cor foi do vermelho até mesmo bem enegrecidas, dependendo da espécie e/ou localização dos indivíduos. Os resultados desta pesquisa concordam com os observados por Naranjo (1947), que também constatou diferentes tonalidades de cor em folhas de diversas culturas sob efeito de geadas em diferentes condiçōes.

Quanto à variação em altura média dos indivíduos mensurados na $1^{\mathrm{a}} \mathrm{e}$ na $2^{a}$ avaliaçōes, algumas espécies apresentam decréscimo em altura (Figura 3), fato que pode ser explicado pelas diferentes formas de avaliação. Enquanto na $1^{\mathrm{a}}$ avaliação consideram-se as alturas totais, dos indivíduos, incluindo até mesmo as partes aparentemente secas, na $2^{\mathrm{a}}$ avaliação consideram-se apenas as alturas das rebrotas, já que muitas mudas haviam perdido suas partes secas e apicais. Nas avaliações posteriores $\left(3^{\mathrm{a}}\right.$ e $\left.4^{\mathrm{a}}\right)$, observou-se o início do processo de recuperação, refletido basicamente na rebrota da maioria dos indivíduos estudados, independente da espécie.

Os resultados referentes a atribuições de notas aos indivíduos no campo (Figura 4), indicaram que a maioria das espécies têm boa capacidade de recupera- 
ção. Nota-se também que os indivíduos localizados nas faixas mais próximas do rio foram os que menos demonstraram os efeitos da geada, ou seja, em todas as avaliações e para todas as espécies, as maiores notas foram atribuídas aos indivíduos desta faixa, o que deve estar associado à ocorrência de maior umidade do ar, que proporciona o aumento da condutibilidade térmica do solo e dificulta 0 resfriamento local. A única exceção foi verificada para $E$. cristagalli, que na $1^{\mathrm{a}}$ e $2^{\mathrm{a}}$ avaliações apresentaram as menores notas nesta localização e, a partir da $3^{\mathrm{a}}$ avaliação apresentou-se praticamente indiferente ao local onde se encontrava. As espécies que apresentaram pior recuperação, quando se considerou a atribuição de notas, foram Alchornea sp., seguida de $H$ courbaril e $P$ regnelli.

A Figura 5, que apresenta as porcentagens médias de indivíduos mortos, indica também que os efeitos das geadas foram menos intensos nas faixas mais próximas do rio, o que pode ser devido a fatores, como porte dos indivíduos localizados neste trecho, em geral maiores, e a presença de indivíduos arbóreos remanescentes, que contribuíram para uma menor perda de calor do solo para a atmosfera. Observou-se também um aumento do número de indivíduos mortos em todas as faixas estudadas, a partir da $2^{a}$ avaliação, o que indicou a necessidade de se efetuarem as avaliações subsequentes.

A análise das figuras 3,4 e 5, indica que a porcentagem média de indivíduos mortos é o parâmetro que mais diferencia as espécies estudadas neste ensaio, mostrando um comportamento diversificado quanto à sensibilidade a geada. Com base nesse parâmetro, verifica-se que as espécies mais resistentes foram I. striata e $E$. cristagalli, enquanto aquelas que apresentaram menor resistência, foram Alchornea sp., $H$. courbaril e $P$. regnelli, devendo ser indicadas com restriçōes para reflorestamento em locais onde geadas ocorrem com mais frequência.

Ainda com relação ao local de ocorrência dos indivíduos e espécies atingidos pela geada, dois aspectos devem ser considerados; aqueles relacionados à ecofisiologia e à distribuição geográfica das espécies. No primeiro caso, sabe-se que as diferentes formas de geada afetam distintamente às plantas, que apresentam sensibilidade variável entre as suas partes. Estruturas subterrâneas são menos resisitentes que as partes aéreas, porém sua resistência é mais uniforme, já que em geral a temperatura dos solos não cai a níveis tão baixos, quando comparada à temperatura do ar. Assim, a rápida formação de brotos e a consequente recuperação dos indivíduos, verificada de forma generalizada nesta pesquisa, pode ser explicada pela proximidade dos brotos ao solo.

Um outro aspecto que deve ser considerado é que, apesar da presença de gramíneas em toda a área estudada, o solo apresenta-se bastante compacto ao redor das mudas, permitindo assim um fluxo de calor por condução térmica para a superfície e, desta forma, proporcionar aquecimento desta e do ar próximo das mudas, atenuando, portanto, os efeitos das geadas sobre as mesmas.

Quanto à distribuição geográfica, Silberbauer-Gottsberger et al. (1977), trabalharam com espécies de cerrado sob efeitos de geadas e verificaram que as espécies cuja principal acorrência se dá em regiōes mais quentes, são aquelas que 



目-Foixal(o-21m dorio) ]-Foixo 2 (21-42m do rio) [] -Foixo 3(42-63mdo rio) 目-Foixo 4 (moior de 63m dorio)

Figura 3 - Alturas médias obtidas das 13 espécies estudadas no experimento, em quatro levantamentos, divididos em faixas de amostragem (Moji-Guaçu - SP. 1990). Alchornea sp. - Al; Cariniana sp. - ca; C. langsdorfii - Cl; Cordia sp. - Co; C. urucurana $\mathrm{Cr}$, C. vecchii - Cy; E. contortisiliquum - En; E. cristagalli - Er; G. americana - Ge; H. courbaril - Hy; I. striata - In; L. muehlbergianus - Lo; P. regnelli - Pl. 

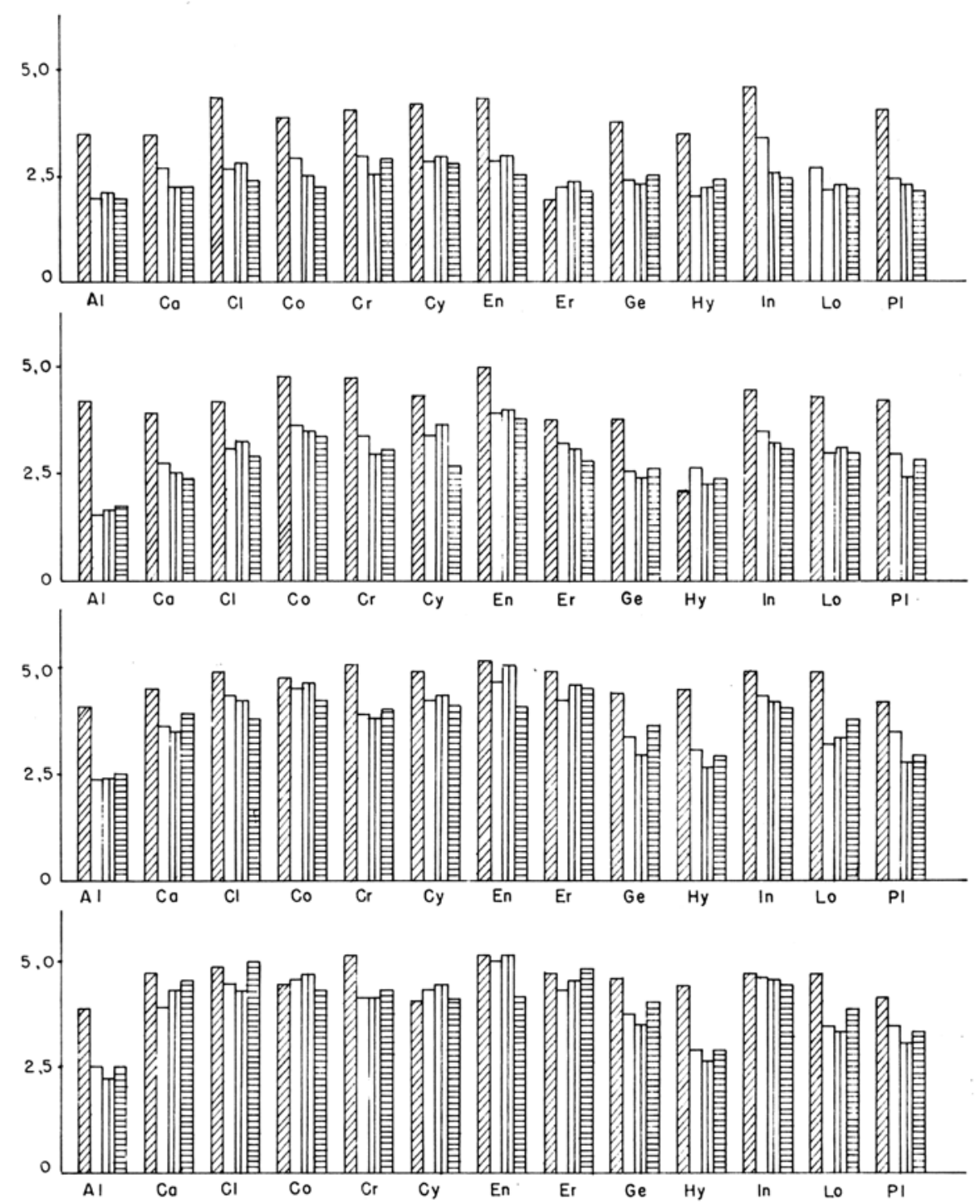

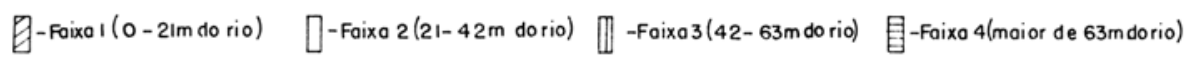

Figura 4 - Notas médias atribuídas para as 3 espécies estudadas no experimento, em quatro levantamentos divididos em faixas de amostragem (Moji-Guaçu - SP. 1990). (1=morta; $2=$ muito afetada; $3=$ mediamente afetada; $4=$ =pouco afetada e $5=$ não afetada). Alchornea sp. - Al; Cariniana sp. - Ca; C. langsdorfii - Cl;Cordia sp. - Co; C. urucurana $\mathrm{Cr}$ C C vechii - Cy; E. contortisiliquum - En; $E$. cristagalli - Er; G. americana - Ge; $H$. courbaril - Hy; I. striata - In; L. muehlbergianus - Lo; P. regnelli - Pl. 

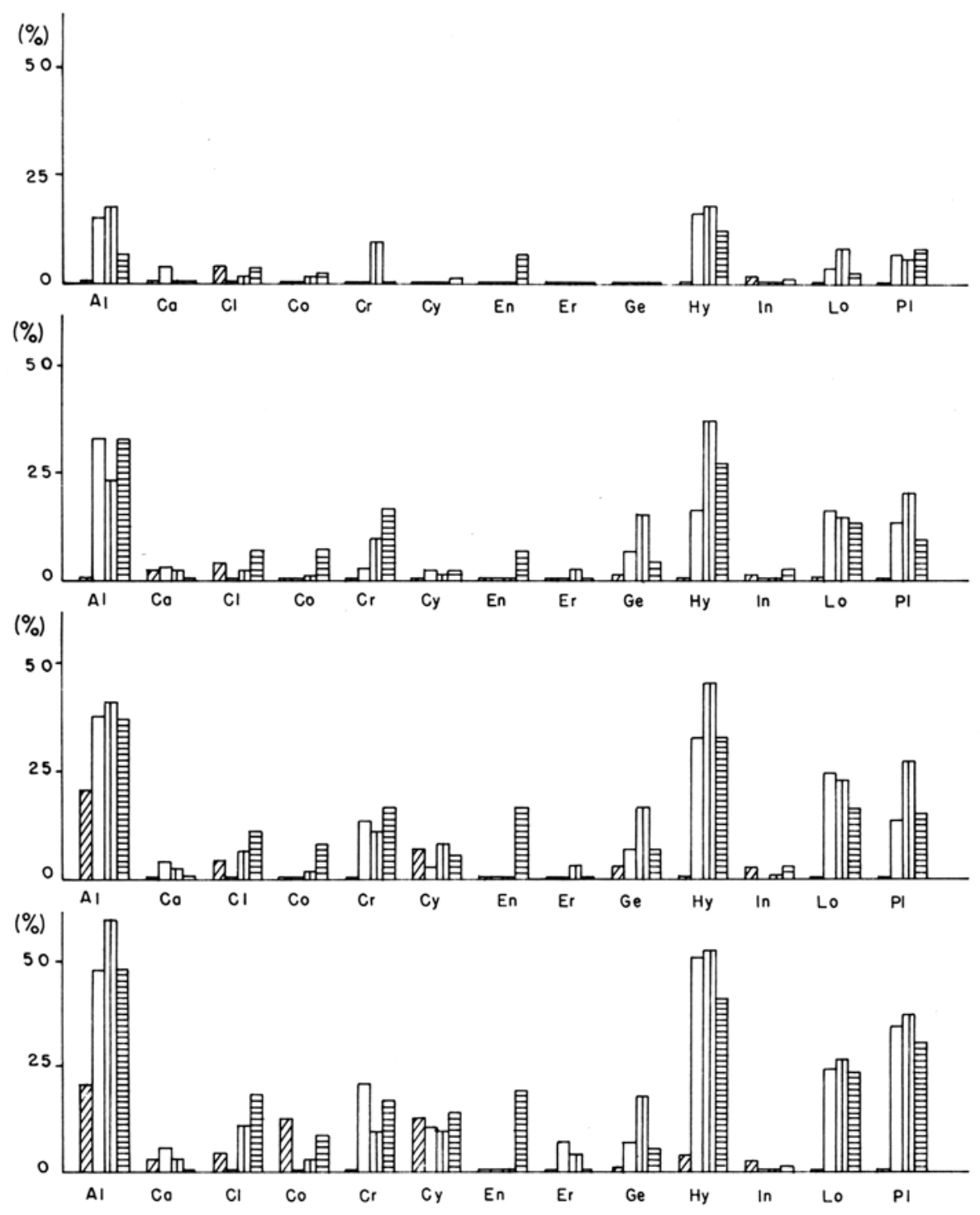

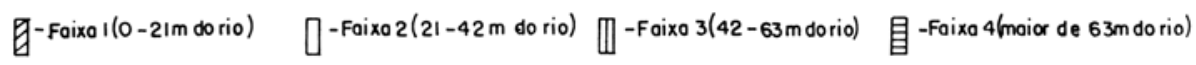

Figura 5 - Porcentagem média de indivíduos mortos das 13 espécies estudadas no experimento em quatro levantamentos divididos em faixas de amostragem (Moji-Gauçu SP. 1990). Alchornea sp. - Al; Cariniana sp. - Ca; C. langsdorfii - Cl; Cordia sp. - Co; C. urucurana - Cr; C. vecchii - Cy; E. contortisiliquum - En; E. cristagalli - Er; G. americana $\mathrm{Ge}$; . courbaril - Hy; I. striata - In; L. muehlbergianus - Lo; P. regnelli - Pl. 
mais sofrem os efeitos das geadas, quando contrapostas com espécies de regiōes mais frias. Os autores verificaram, ainda, que existe uma correlação entre os efeitos da geada, não apenas sobre a distribuição geográfica, mas também sobre a frequência das espécies estudadas na área.

No caso desta pesquisa, a maioria das espécies estudadas são de ampla distribuição geográfica, especialmente pelo fato de serem espécies típicas de matas de galeria,variando apenas em termos de frequência.

Conclusivamente, o que se pode adiantar, com base nos resultados obtidos nesta pesquisa, é que das 13 espécies estudadas, apenas P. regnelli, H. courbaril e Alchornea sp. apresentam restrições a sua utilização em regiōes sujeitas à geadas periódicas, enquanto que as demais espécies apresentam grande capacidade de recuperação.

\section{Referências bibliográficas}

BARBOSA, L.M. 1989 Estudos interdisciplinares do Instituto de Botânica em Moji-Guaçu, SP. In: Anais do Simpósio sobre Mata Ciliar. São Paulo. p.171191.

BARBOSA. L.M.; L.M, BARBOSA; R. ANDREANI JR.; T.S. SILVA, S.A. VERONESE, \& M.F.B. ZELLER. 1989a. Estudos dos efeitos da periodicidade de inundação sobre o vigor das sementes e desenvolvimento de plântulas para oito espécies ocorrentes em mata ciliar. In: Anais do Simpósio sobre Mata Ciliar. São Paulo. p.310-319.

BARBOSA, L.M.; J.M. BARBOSA; E.A. BATISTA; W. MANTOVANI; S.A. VERONESE, \& R. ANDREANI JR.; 1989b. Ensaios para estabelecimentos de modelos para recuperação de áreas degradadas de matas ciliares, MojiGuaçu (SP) - Nota Prévia. In: Anais do Simpósio sobre Mata Ciliar. São Paulo. p.268-283.

DEMATTÊ, M.E.S.P. 1989. Recomposição de matas ciliares na região de Jaboticabal, SP. In: Anais do Simpósio sobre Mata Ciliar. São Paulo. p.160170.

MANTOVANI, W. 1989. Conceituação e fatores condicionantes. In: Anais do Simpósio sobre Mata Ciliar. São Paulo. p.11-19.

MARQUES, J.Q. de A.; J. BERTONI, \& G.B. BARRETO, 1961. As perdas por erosão no Estado de São Paulo. Bragantina, Campinas, SP, 20(47):11431181.

NARANJO, P.V.1947. La helada y la necrosis fria de las plantas.Boletim del Instituto Botânico de la Universidad Central, Ecuador, 6(7):55-170.

PORTO, M.L.; H.M. LONGHI; V. CITADINI; R.F. RAMOS, \& J.E. de A. MARIATH. 1976. Levantamento fitossociológico em áreas de uma "mata de baixio" na 
Estação Experimental de Silvicultura Tropical - INPA - Manaus, AM. Acta Amazônica, 6(3):301-318.

SALVADOR, J.L.G. 1987. Considerações sobre as matas ciliares e a implantação de reflorestamento misto nas margens de rios e reservatórios. SP, CESP. 29 p.

SILBERBAUER-GOTTSBERGER, I.; W. MORAWETZ \& G. GOTTSBERGER, 1977. Frost damage of Cerrado plants in Botucatu, Brazil, as related to the geographical distribution of the species. Biotropica, Costa Rica, 9(4):253261.

STRUFFALDI DE VUONO, Y.; L.M. BARBOSA, E.A. BATISTA, \& O.A. GURGEL FILHO. 1982. Efeitos biológicos da geada na vegetação do cerrado. In: Anais do Congresso Nacional sobre Essências Nativas. Campo do Jordão, SP. Silvicultura em São Paulo, 16A(1):545-547. 